Review

\title{
Nano-Assembly Small Molecule Probe - New Horizon for Molecular Imaging
}

\author{
Baozhong Shen \\ ${ }^{1}$ Molecular Imaging Research Center (MIRC), Harbin Medical University, Harbin, Heilongjiang, China. \\ ${ }^{2}$ TOF-PET/CT/MR Center, the Fourth Hospital of Harbin Medical University, Harbin, Heilongjiang, China. \\ Corresponding author. E-mail: shenbzh@vip.sina.com
}

Received: Dec. 23, 2017; Accepted: Dec. 27, 2017; Published: Dec. 31, 2017.

Citation: Baozhong Shen, Nano-Assembly Small Molecule Probe - New Horizon for Molecular Imaging. Nano Biomed. Eng., 2017, 9(4): 355-363. DOI: 10.5101/nbe.v9i4.p355-363.

\begin{abstract}
Molecular imaging allows the biological processes taking place in the body to be viewed at a cellular and molecular level. Molecular imaging probe is used to visualize, characterize and quantify the processes. Nano-assembly small molecule probe provides an attractive means to precisely engineer nanomedicine with distinct biophysicochemical properties and simplify the optimization of formulation, which is an emerging and promising area that can integrate the advantages of the two kinds of molecular imaging probes with high loading capacity, effective signal amplification, preferable clearance, etc. Herein, we review main advances such as small molecules self-assembly into nanoprobes, its development and application, including fluorescent, magnetic resonance imaging, and nuclide marker labeled nano-assembly small molecules probes, as well as "smart" probes of this kind and their applications in molecular imaging.
\end{abstract}

Keywords: Molecular imaging; Self-assembly; Small molecule probe; Nanodrug

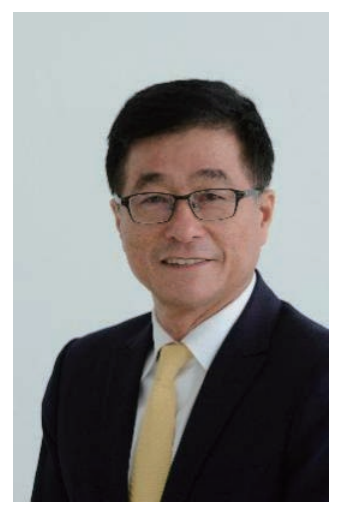

Baozhong Shen, M.D.,Ph.D. Professor of Medical Imaging and Nuclear Medicine. President of the Fourth Hospital of Harbin Medical University. He has been working 33 years in the field of medical imaging. He established the first molecular imaging center in China in 2002, and completed some clinical translation of a number of basic research achievements from bench to bedside. He edited the first molecular imaging monograph "Molecule Imaging"(People's Health Publishing House) in China. He won the second prize of National Science and Technology Progress Award as the first accomplisher. $\mathrm{He}$ is the first doctoral supervisor in medical imaging and nuclear medicine major in Heilongjiang Province and had trained more than 130 postdoctoral fellows, doctoral and postgraduate students, besides trained a large number of molecular imaging professionals with multidisciplinary professional knowledge.

\section{Introduction}

Molecular Imaging (MI) enables the visualization of the cellular function and the follow-up of the molecular process in living organisms without perturbing them [1]. It can realize noninvasive and real-time visualization, measurement of physiological or pathological process in the living organism at the cellular and molecular level, providing an effective method of information acquiring for diagnosis, therapy, 
drug development and evaluating treatment efficacy [2]. Molecular imaging differs from traditional imaging in that it can image fine molecular changes which opens up an incredible number of exciting possibilities for medical applications [3-6]. Molecular imaging requires high resolution and high sensitive instruments and specific molecular imaging probes that link the imaging signal with molecular event [7-9]. The pioneer scientists had pointed out the prerequisites for MI probe to meet the successful molecular imaging [10], such as: (a) availability of high-affinity probes with reasonable pharmacodynamics; (b) the ability of these probes to overcome biologic delivery barriers (vascular, interstitial, cell membrane); (c) use of amplification strategies (chemical or biologic); and (d) availability of sensitive, fast, high-resolution imaging techniques. Those principles should guide us when design a new probe for MI. Recently, the application of new emerging chemical technology and nanotechnology has stimulated the development of molecular imaging probes. This review is intended as a short primer on the subject of amphiphilic small molecules without carriers, which directly to form nano delivery systems that can significantly improve the shortages and disadvantages of traditional molecular imaging probes.

\section{The Advantages of Nano-assembly Small Molecules Probe for MI}

The field of nano MI probes has been developing worldwide and bring many solutions to modern medicines limitations in molecular imaging and drug delivery, etc. [11-14]. The main challenges in nanomedicine involve efforts to improve cargo loading efficiency, pharmacokinetics, live imaging, biosafety and ultimately clinical translation $[15,16]$. The concern of using nanostructured MI probes lie in three aspects. First, their drug carrying capacity is very low, typically below $5 \%(\mathrm{w} / \mathrm{w})$ [17]. Second, there is an inherent difficulty to control and characterize the amount of drugs loaded in each particle. There will also be a loading variation from particle to particle, and as such the loading capacity only represents the averaged amount of drug loaded in the whole system. Finally, the long term toxicity of these synthetic nanoobjects remains unclear [18].

Small molecules, of which the size is usually less than 500Da, are playing an important role in molecular imaging. Due to their small size, small molecules have a wide range of application including intracellular and central nervous system [19]. Molecular assembly of small molecules into nanoprobe is an emerging area in the MI field. By incorporating a small molecule(such as drug) as one component of an amphiphilic monomer, the drug becomes part of its own delivery vehicle a nano-sized entity that possess a well-defined shape and distinct physicochemical properties different to those of the small molecules [20]. Self-assembly of nanostructures provides an attractive means by which to precisely engineer nanomedicines with distinct biophysicochemical properties, thus simplifying the optimization of formulation. Besides, self-assembled nanostructures are of particular interest because they can be readily prepared to assume a variety of sizes and shapes with adjustable surface chemistry through solution state assembly processes. After small molecules realized nano-assembly, their detection signal can be effectively amplified. A further attraction is that these supramolecular carriers can dissociate into individual molecules that could be removed through renal clearance once their desired function is accomplished [21].

The above leading to the fact that self-assembled small molecule vehicle have a high loading capacity (up to $100 \%$ if the nanostructures are composed of free molecules), also allow quantitative control of the molecules so that the assembled nanostructures have the same content as a single molecule; and can minimize the toxicity caused by additional synthetic carriers [22, 23]. In addition, the nanoscale features of amphiphilic small molecule assembles can effectively overcome the multidrug resistance (MDR) (20) of tumor cells, have enhanced permeability and retention (EPR) characteristics [24, 25], which is not applied to small molecules because of their rapid diffusion into the circulating blood followed by renal clearance, and can escape the reticuloendothelial system (RES). If the small molecules could exhibit nanoscale characteristics by themselves without the help of nano vehicles, a promising self-delivery system integrating both the advantages of free molecules and nanocarriers could be expected. By incorporating a small molecule (such as drug) as one component of an amphiphilic monomer, the small molecules becomes part of its own delivery vehicle - a nano-sized entity that possess a welldefined shape and distinct physicochemical properties different to those of the small molecules. This strategy had received significant success in the drug development filed, and this application and benefit not 
limited to the drug, which also can be promoted to MI probe design and fabrication.

\section{The Process of Small Molecules Self-Assembly into Nano Structure}

At the molecular level, self-assembly involves competition between intermolecular (or thermodynamic) forces. The forces are mainly Van der Waals attraction and electrostatic repulsion, the former motivating agglomeration in order to minimize the free energy of the system, and the latter stabilizing nanoparticles by repulsion of surrounding NPs. When these forces balance, agglomeration stops such that NPs are successfully self-assembled.

The self-assembly of small molecules into discrete nanostructures has rapidly become a valuable tool for the creation of functional materials since the advent of supramolecular chemistry. Through the spontaneous association of appropriately designed monomers, nano-sized entities can be constructed that possess a well-defined shape and distinct physicochemical properties. Molecular self-assembly is a spontaneous association of individual small molecular components into well-ordered structures assisted by non-covalent interactions, such as the hydrogen bond interaction, pi-pi interaction, hydrophobic interaction, and charge interaction [26]. The morphology of these assembled structures is determined by the constituent parts of the monomer, which by necessity is often amphiphilic in nature and therefore composed of hydrophilic and hydrophobic domains. Hydrophilic segments, for example, range from simple charged (phosphate, ammonium, carboxylate) and neutral (oligo-ethylene glycol, oligosaccharides) groups to more complex oligo-peptides, and peptide nucleic acids. Hydrophobic segments include linear hydrocarbons, simple and extended aromatic systems, etc. [20]. A promising application of this molecular assembly approach is for the creation of biologically active materials, with the delivery of functional constitute being a particular avenue of development.

\section{The Development of Nano-Assembly Small Molecules in Drug Field}

In a variety of cancer treatments, chemotherapy is an indispensable choice for most cancer cases because of its high efficiency. Unfortunately, due to the small molecular weight of free anticancer drugs, traditional chemotherapy has several limitations, including poor bioavailability, rapid blood/kidney clearance, nonspecific selectivity, low tumor build-up, severe drug resistance, and adverse effects on health. In order to overcome these obstacles, there is a need for an effective drug delivery system for cancer therapy.

Over the past years, the use of nanoscale carriers to modify the drugs pharmacokinetic properties and biodistribution profiles has been the primary focus of research in the drug delivery community [25]. Besides the first-line chemotherapy drugs such as paclitaxel, doxorubicin, fluorouracil, and gemcitabine, there is a class of recombinant chimeric polypeptide (CP) nanoparticles for the delivery of chemotherapeutics to solid tumors has been developed according to the attractive features of drug-loaded nanoparticles [27]. CPs are comprised of two components: (1) a hydrophilic elastin-like polypeptide (ELP)domain consisting of repeats of the pentapeptide Val-ProGly-Xaa-Gly, where Xaa is any amino acid except Pro, and (2) a C-terminal C(GGC) peptide segment that provides eight unique cysteine residues that can be used as sites for drug attachment. Conjugation of copies of the chemotherapeutic doxorubicin (Dox) to the C-terminal drug attachment domain through an acid-labile linker results in the spontaneous selfassembly of $\sim 40 \mathrm{~nm}$ diameter spherical micelles within which the drug is sequestered. The efficacy of these CP-Dox nanoparticles in the C26 murine colon carcinoma model were demonstrated, where $\sim 90 \%$ of a tumor bearing cohort was cured following a single injection. This drug delivery vehicle succeeded in enhancing doxorubicin's accumulation in the primary tumor formulation and would be a more effective neoadjuvant agent compared to freely dissolved drug.

Such strategy had been applied into synthesize the hydrophilic anticancer drug irinotecan (Ir) and the hydrophobic anticancer drug chlorambucil (Cb) through the hydrolyzable ester bond to the amphiphilic drug-drug (ADDC) [28]. Amphoteric Ir-Cb conjugates are free to assemble nanoparticles into water and have a longer blood retention half-life compared to free drugs, which contributes to the accumulation of drugs in tumor tissue and promotes their cellular uptake. The benefits of nanoscale features of Ir-Cb ADDC nanoparticles are effective in overcoming the multidrug resistance of tumor cells. After intracellularization, the ester bonds between the hydrophilic and hydrophobic 


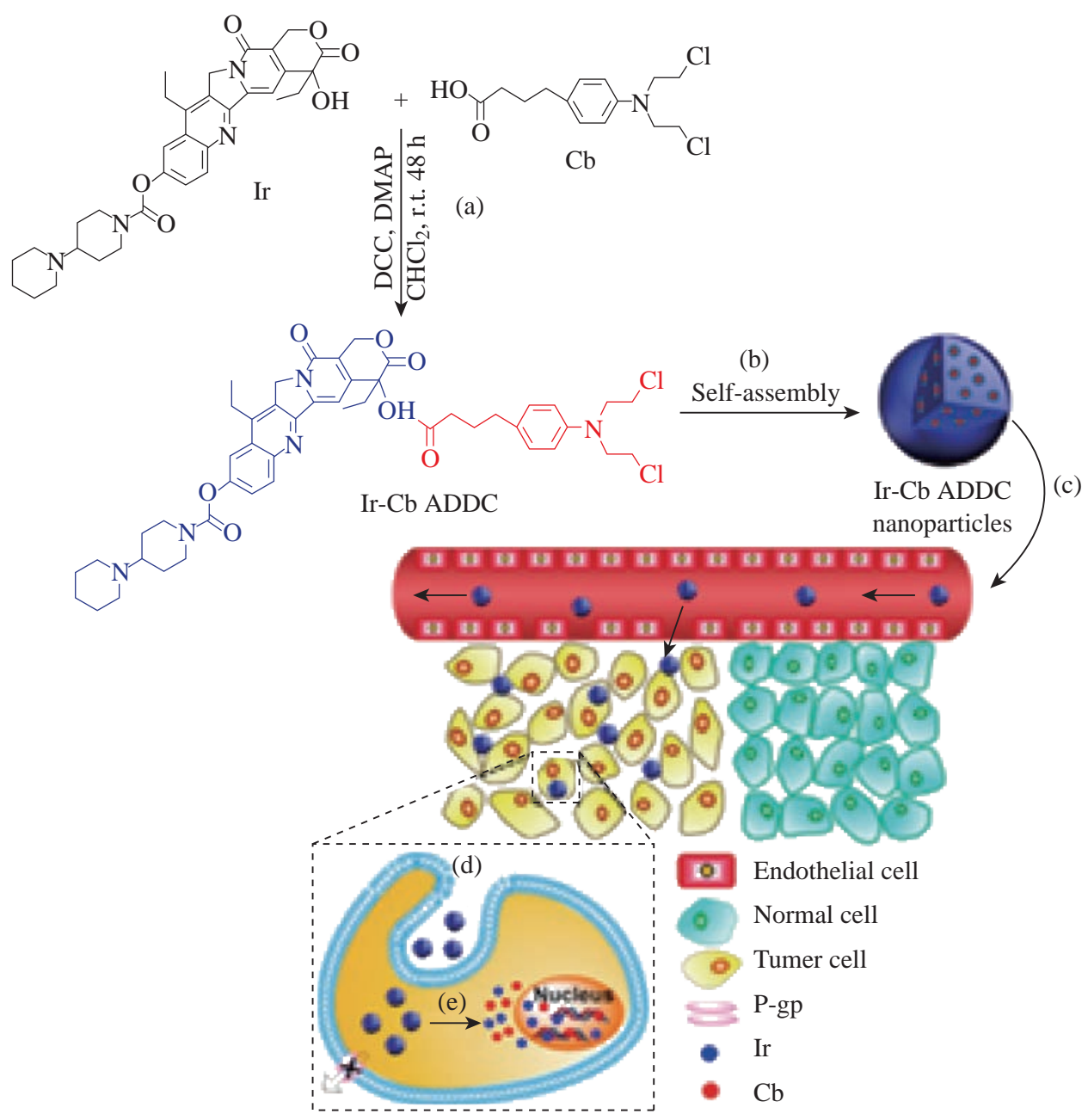

Fig. 1 Schematic diagram of amphiphilic drug-drug conjugate (ADDC) from fabrication, self-assembly to self-delivery. (a) Synthesis of Ir-Cb ADDC through esterification in DCC/DMAP-catalyzed system. (b) The Ir-Cb ADDC self-assembles into nanoparticles in water. (c) Passive tumor targeting is achieved by the advantage of the EPR effect, which facilitates the Ir-Cb ADDC nanoparticles to access tumors by way of their leaky vasculature. (d) The Ir-Cb ADDC nanoparticles enter tumor cells by endocytosis. (e) Ir and Cb are released by the cleavage of the ester bond in tumor cells and then diffuse into nucleus [28].

drugs undergo hydrolysis to release free Ir and Cb, resulting in excellent anticancer activity in vitro and in vivo. the ADDC strategy was described in Fig. 1 here.

Such strategy has been relatively widely explored through diverse synthetic compounds in drug development. For the EPR effect does not apply to lowmolecular-weight drugs because of their rapid diffusion into the circulating blood followed by renalclearance. One advantage of the strategies was to synthesize an amphiphilic copolymer comprised of hydrophilic part and hydrophobic fragment that facilitates rapid selfassembly into nanomicelles suitable for passive tumor targeting by the EPR effect. For the single hydrophobic or hydrophilic molecules alone cannot self-assemble into stable nanoparticles, requiring amphiphilic or ionic materials to support nanoparticle stability and function in vivo. The developed drug included conjugating of hydrophobic drugs with short peptide segment, conjugating of hydrophobic drugs with hydrophilic drugs, etc. [25, 29, 30].

\section{The Development of Nano-Asse- mbly Small Molecules in MI Field Fluorescent marker labeled nano-assembly small molecule MI probe}

Indocyanine green (ICG) is a near-infrared (NIR) imaging and diastereomer for laser-mediated photothermal therapy. This new feature is mainly used for integrated optical imaging and photothermal therapy. However, ICG applications are subject to concentration-dependent aggregation, water instability, non-specific binding to proteins, and target-specific deletions. In order to overcome these limitations, a 
novel ICG-containing nanostructure was designed by noncovalent self-assembly chemistry between phospholipid-polyethylene glycol (PL-PEG) and ICG. The in vitro experiments show that intrinsic ICGPL-PEG can be used for cell imaging and selective photothermal cell destruction. This novel ICG-PL-PEG nanostructures can be used as multifunctional probes for cancer diagnosis and treatment when combined with other therapies and imaging agents. Based on the characteristics of ICG-PL-PEG, it is expected that the self-assembly process of this probe follows the scheme outlined in Fig. 2 [31].

\section{MR marker labeled nano-assembly small molecule MI probe}

Among the current imaging modalities, MRI is a unique tool to investigate disease non-invasively. The advantages of MRI are its excellent soft tissue contrast, diffusion-weighted imaging, dynamic contrast-enhanced imaging, functional MRI and others personalized sequences as well as MR spectroscopy with the quantitative physiologic information. With the development of MRI, scientists developed some novel MR molecular probes. As a proof-of-concept, Ma's team designed amphiphilic small molecule consisting of water-soluble MRI contrast agent Gd(DTPA), diethylene triamine pentaacetic acid and waterinsoluble anticancer drug camptothecin (CPT) (Scheme 1(a)). Gd(DTPA) is one of the most commonly used contrast agents to enhance the tissue contrast of MRI. In addition, CPT is a topoisomerase I(topol) enzyme inhibitor to induce replication/ transcription- mediated DNA damage. The small molecular theranostic nanoparticles are prepared through the aqueous selfassembly of an esterase-responsive amphiphilic molecule Gd(DTPA-CPT), in which Gd(DTPA) is conjugated with CPT through esterification. After injected intravenously, Gd(DTPA-CPT) nanoparticles can accumulate in tumor tissues via passive targeting mechanism (Scheme 1b). Once internalized by cancer cells, Gd(DTPA-CPT) nanoparticles will decompose and release free CPT via ester hydrolysis catalyzed by esterase in the cytoplasm. On the other hand, dissociation of the redundant Gd(DTPA-CPT) nanoparticles in blood circulation would be accelerated because their concentration decreases below the critical aggregation concentration (CAC), resulting in effective clearance from the kidneys and low longterm toxicity. Thanks to the rapid equilibrium shift with small molecules, these nanoparticles would open new avenues for exploration of nano DDS, which integrates with obvious anticancer effect and low-toxic metabolism pathway for clinical applications [32].

\section{Nuclide labeled nano-assembly small molecule MI probe}

PET serves as a highly sensitive imaging tool to assist in the development of nanoprobes to realize the potential of nanomedicine for early-stage detection, diagnosis, treatment, and monitoring of disease progression, regression, and recurrence.

Mutated epidermal growth factor receptor (EGFR) is an important biomarker for cancer diagnosis and molecular target for many anticancer drugs. Localizing EGFR and evaluating EGFR mutational status can help to identify patients who are potentially the most suitable ones for targeted treatments. Increasing attention has been paid onto the use of radionuclideslabeled small molecule TKIs to identify EGFR mutation in vivo. The major problem is that most of the tracersare lipophilic and can be rapidly eliminated through hepatobiliary clearance. As a result, the level of radionuclides-labeled small molecule TKI up-taken

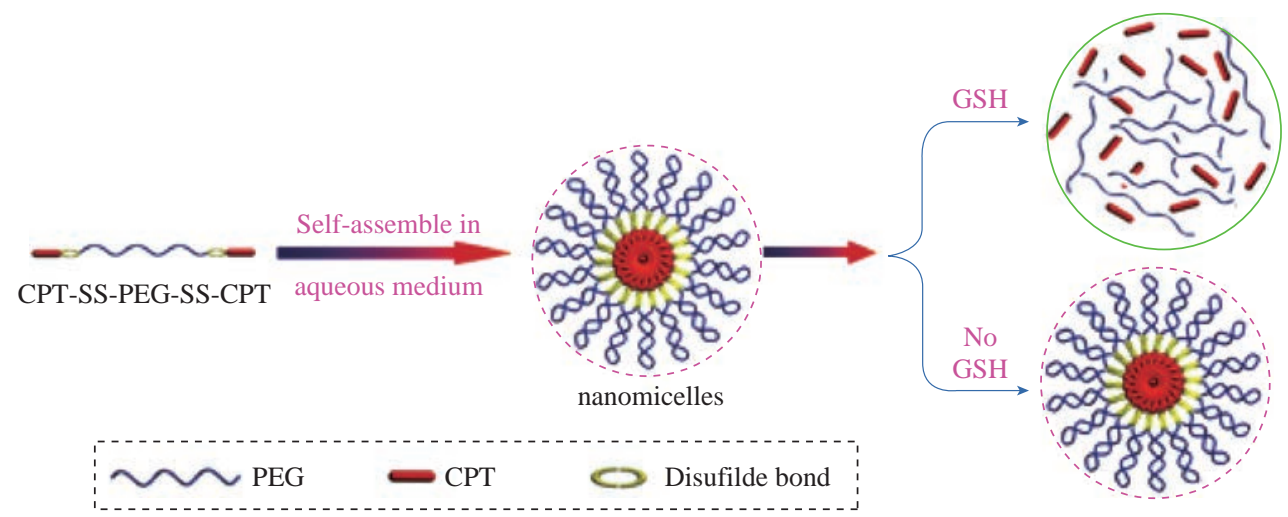

Fig. 2 Schematic outline of the predicted self-assembly behavior of CPT-SS-PEG-SS-CPT under aqueous conditions and its drug release behavior [29]. 
by tumor tissues is too low to generate an adequate target-to-background ratio of PET-detectable signals.

Our team developed a novel nano-assembly amphiphilic small molecules probe modified from EGFR tyrosine kinase inhibitor2-(2-(2-(2-(4-(3-chloro4-fluorophenylamino)-6-methoxyquina, MPG) labeled with ${ }^{99 \mathrm{~m}}$ Tcor $^{18} \mathrm{~F}$ and evaluated its EGFR binding capacity from bench to bedside [33, 34]. A simple and high-efficiency method for the synthesis of nuclide labeled MPG was developed, in which we optimized the conditions producing the new probe, and evaluated its chemical and biological characteristics in vitro and in vivo.

These amphiphilic small molecules self-assembled ${ }^{18} \mathrm{~F}$-MPG nanoparticles are around $30 \mathrm{~nm}$ in diameter. Compared with the commonly used ${ }^{18}$ F-FDG in tumor

(a)

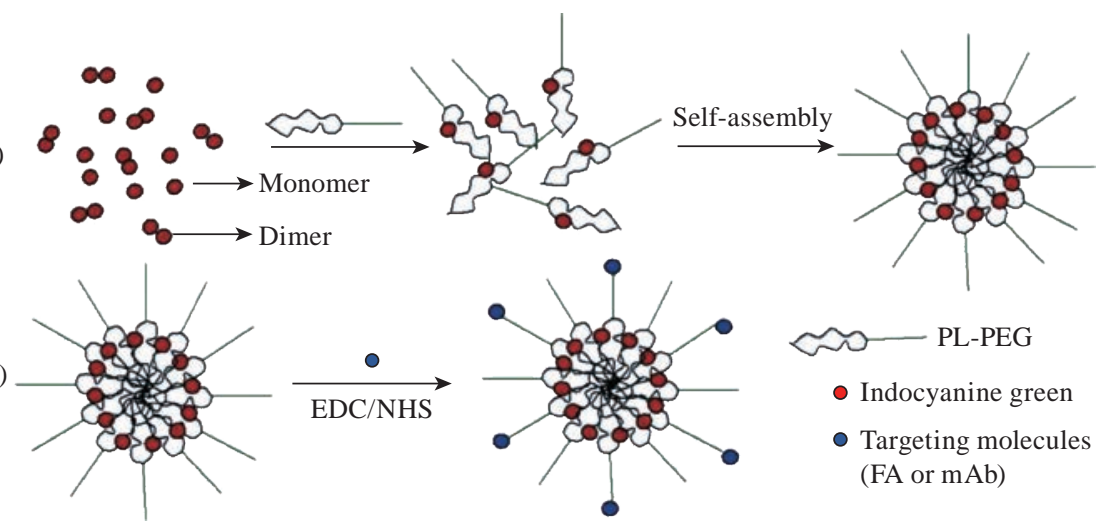

Fig. 3 (a) Self-assembly process of ICG-PL-PEG probe. (b) Targeted modification of ICG-PL-PEG probe.FA or mAb was linked on the surface via amidation reaction. EDC: 1-ethyl-3-(3-(dimethylamino)propyl) carbodiimide. NHS: N-hydroxysulfosuccinimide [31].

(a)

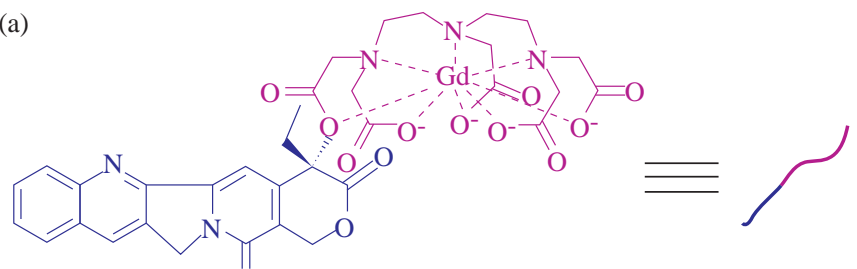

$\mathrm{O}$

Gd-DTPA-CPT

Self-assembly

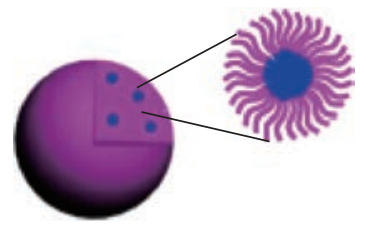

Gd-DTPA-CPT Nanoparticles

(b)

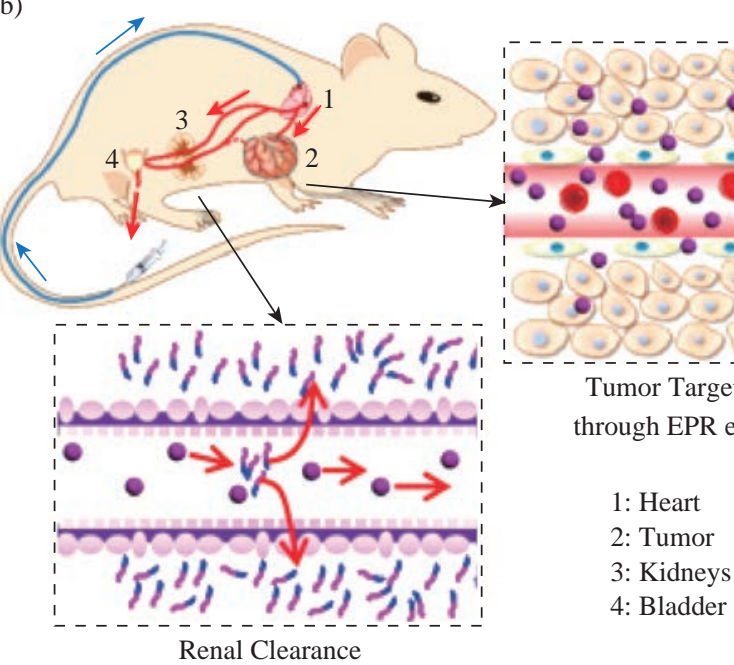

Fig. 4 Gd(DTPA-CPT) nanoparticles for tumor targeting and renal excretion. (a) Schematic illustration of amphiphilic Gd(DTPACPT) self-assembly into nanoparticles. (b) Scheme of in vivo passive tumor targeting and renal clearance of Gd(DTPA-CPT) nanoparticles [32]. 

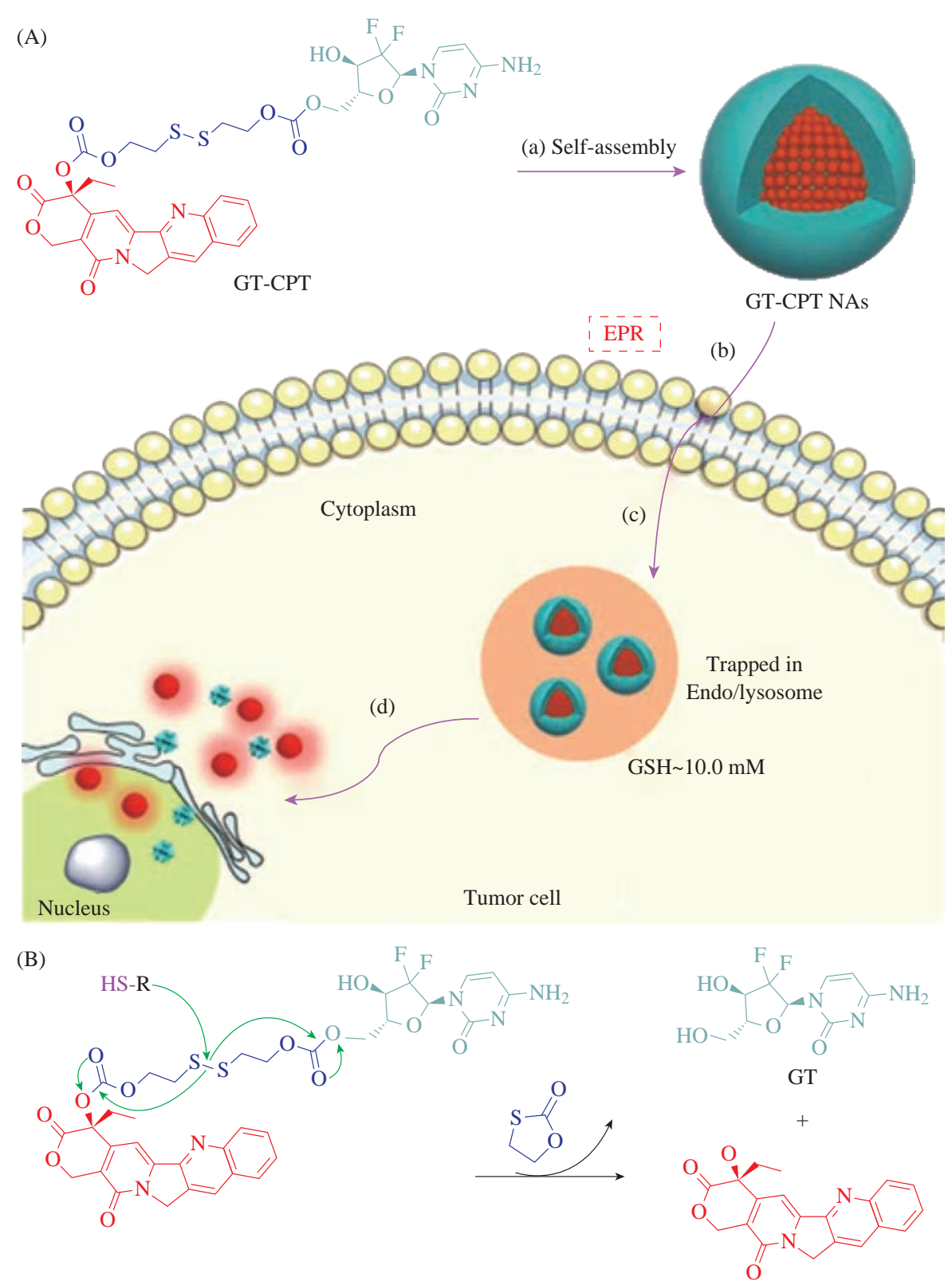

GT-CPT prodrug

CPT

Fig. 5 (A) Schematic diagram of amphiphilic GT-CPT prodrug for combination chemotherapy and reduction-responsive drug release: (a) the GT-CPT NAs are formed by self-assembly; (b) the GT-CPT NAs are internalized under enhanced permeability and retention (EPR) effect; (c) the GT-CPT NAs are trapped in endo/lysosomes; and (d) the GT-CPT NAs self-decompose and release dual drugs into cell nucleus. (B) The proposed mechanism of drug release induced by reducing agent.

patients, ${ }^{18} \mathrm{~F}-\mathrm{MPG}$ has no uptake in normal brain tissue and it is superior to ${ }^{18} \mathrm{~F}$-FDG in mutant EGFR targeted molecular imaging. These small molecule radiopharmaceuticals, designed to specifically track the extent and location of lung cancers through detecting mutated EGFR, are likely manufactured on demands in a local radiopharmacy and capable of improving patient management and enhancing clinicians' ability to select the most suitable patients to apply moleculetargeting drugs.

\section{The "Smart" Nano-Assembly Small Molecules Probe}

We refer "Smart" here to the nano-assembly structures which can response to the exterior environment, such as $\mathrm{pH}$ sensitivity, enzyme responsiveness, etc. [35]. Hydrophilic gemcitabine and hydrophobic camptothecin are linked by disulfide bonds and abbreviated as GT-CPT. The obtained amphiphilic prodrug conjugate can self-assemble 
into nanoparticles in water and exhibit strong micelle stability and excellent hemocompatibility in vivo. GT-CPT prodrug conjugate can achieve up to $~ 75$ wt\% accurate drug loading, showing an unsupported effective drug delivery model. In addition, the reduced reaction disulfide bonds are capable of controlling drug release in the presence of a tumorspecific microenvironment. It was found that each of these heterozygous drug components (CPT and GT) showed not only enhanced cytotoxicity alone but also a significant synergistic effect on HeLa and MCF-7 cancer cells. This stimulating reactive prehybridization of drug conjugates for the efficient co-delivery of multiple anticancer chemotherapeutic agents, which can stimulate the use of this mixed prodrug conjugate for combination chemotherapy. Furthermore, the disulfide linker was able to breakdown and release the drugs in response to the highly reductive microenvironment resembling tumor site (Fig. 3) [36, 37].

\section{Conclusions}

The nanoscale features of amphiphilic small molecule probes have enhanced stability, extended circulation lifetime, site-specific targeting, improved MI efficacy, and other advantages, integrating characteristics of nanoparticles and small molecules. However, nano-assembly small molecules probes also have their own shortcomings and deficiencies, such as still accumulate in other organs similar to conventional nanoparticles, may lead to long-term toxicity, etc. Detailed studies on MI mechanism, and emphasize the clinical translation and benefits will further brighten the promising field.

\section{Acknowledgments}

This work was supported by the National Basic Research Program of China (2015CB931800), National Natural Science Foundation of China (81627901, 31210103913), and the Key Laboratory of Molecular Imaging Foundation (College of Heilongjiang Province).

\section{Conflict of Interests}

The authors declare that no competing interest exists.

\section{References}

[1] R. Weissleder, Molecular imaging: Exploring the next frontier. Radiology, 1999, 212(3): 609-614.

[2] S.S. Gambhir, Molecular imaging of cancer with positron emission tomography. Nature Reviews Cancer, 2002, 2(9): 683-693.

[3] M. Rudin, R. Weissleder, Molecular imaging in drug discovery and development. Nature Reviews Drug Discovery, 2003, 2(2): 123-131.

[4] T.F. Massoud, S.S. Gambhir, Molecular imaging in living subjects: seeing fundamental biological processes in a new light. Genes \& Development, 2003, 17(5): 545-580.

[5] S.A. Wickline, G.M. Lanza, Nanotechnology for molecular imaging and targeted therapy. Circulation, 2003, 107(8): 1092-1095.

[6] X. Chen, Multimodality imaging of tumor integrin alphavbeta3 expression. Mini-reviews in Medicinal Chemistry, 2006, 6(2): 227-233.

[7] 7. D. Pan, S.D. Caruthers, A. Senpan, et al., Revisiting an old friend: Manganese-based MRI contrast agents. Wiley Interdisciplinary Reviews-nanomedicine and Nanobiotechnology, 2011, 3(2): 162-173.

[8] X. Yang, Interventional Molecular Imaging. Radiology, 2010, 254(3): 651-654.

[9] R. Weissleder, M.C. Schwaiger, S.S. Gambhir, et al., Imaging approaches to optimize molecular therapies. Sci Transl Med., 2016, 8(355): 355ps16.

[10] R. Weissleder, U. Mahmood, Molecular imaging. Radiology, 2001, 219(2): 316-333.

[11] G. Chen, H. Qiu, P.N. Prasad, et al., Upconversion nanoparticles: design, nanochemistry, and applications in theranostics. Chemical Reviews, 2014, 114(10): 51615214.

[12] C. Li, A targeted approach to cancer imaging and therapy. Nature Materials, 2014, 13(2): 110-115.

[13] Y. Chen, G. Liang, Enzymatic self-assembly of nanostructures for theranostics. Theranostics, 2012, 2(2): 139-147.

[14] D. Pan, G.M. Lanza, S.A. Wickline, et al., Nanomedicine: Perspective and promises with ligand-directed molecular imaging. European Journal of Radiology, 2009, 70(2): 274-285.

[15] M. Ferrari, Cancer nanotechnology: Opportunities and challenges. Nature Reviews Cancer, 2005, 5(3): 161-171.

[16] A.S. Thakor, S.S. Gambhir, Nanooncology: The future of cancer diagnosis and therapy. CA: A Cancer Journal for Clinicians, 2013, 63(6): 395-418.

[17] H. Lu, J. Wang, T. Wang, et al., Recent progress on nanostructures for drug delivery applications. Journal of Nanomaterials, 2016, 2016: 20.

[18] H. Chen, W. Zhang, G. Zhu, et al., Rethinking cancer nanotheranostics. Nat Rev Mater., 2017, 2: 17024.

[19] Z. Chen, Y. Wang, Y. Lin, et al., Advance of molecular imaging technology and targeted imaging agent in imaging and therapy. BioMed Research International, 2014, 2014: 819324.

[20] A.G. Cheetham, P. Zhang, Y. Lin, et al., Synthesis and self-assembly of a mikto-arm star dual drug amphiphile containing both paclitaxel and camptothecin. Journal of Materials Chemistry B., 2014, 2(42): 7316-7326.

[21] L.L. Lock, M. Lacomb, K. Schwarz, et al., Self-assembly of natural and synthetic drug amphiphiles into discrete supramolecular nanostructures. Faraday Discussions, 2013, 166(0): 285-301.

[22] A.G. Cheetham, P. Zhang, Y. Lin, et al., Supramolecular nanostructures formed by anticancer drug assembly. Journal of the American Chemical Society, 2013, 135(8): 2907-2910. 
[23] Y. Shen, E. Jin, B. Zhang, et al., Prodrugs forming high drug loading multifunctional nanocapsules for intracellular cancer drug delivery. Journal of the American Chemical Society, 2010, 132(12): 4259-4265.

[24] A.K. Iyer, G. Khaled, J. Fang, et al., Exploiting the enhanced permeability and retention effect for tumor targeting. Drug Discovery Today, 2006, 11: 812-818.

[25] Y. Wang, D. Liu, Q. Zheng, et al., Disulfide bond bridge insertion turns hydrophobic anticancer prodrugs into selfassembled nanomedicines. Nano Letters, 2014, 14(10): 5577-5583.

[26] C. Ren, J. Zhang, M. Chen, et al., Self-assembling small molecules for the detection of important analytes. Chemical Society Reviews, 2014, 43(21): 7257-7266.

[27] E. Mastria, M. Chen, J.R. Mcdaniel, et al., Doxorubicinconjugated polypeptide nanoparticles inhibit metastasis in two murine models of carcinoma. Journal of Controlled Release, 2015, 208: 52-58.

[28] P. Huang, D. Wang, Y. Su, et al., Combination of small molecule prodrug and nanodrug delivery: Amphiphilic drug-drug conjugate for cancer therapy. Journal of the American Chemical Society, 2014, 136(33): 11748-11756.

[29] X. Li, H. Wen, H. Dong, et al., Self-assembling nanomicelles of a novel camptothecin prodrug engineered with a redox-responsive release mechanism. Chemical Communications, 2011, 47(30): 8647-8649.

[30] X. Zheng, Z. Li, L. Chen, et al., Self-assembly of porphyrin-paclitaxel conjugates into nanomedicines: Enhanced cytotoxicity due to endosomal escape. Chemistry-an Asian Journal, 2016, 11(12): 1780-1784.

[31] X. Zheng, D. Xing, F. Zhou, et al., Indocyanine greencontaining nanostructure as near infrared dual-functional targeting probes for optical imaging and photothermal therapy. Molecular Pharmaceutics, 2011, 8(2): 447-456.

[32] Y. Ma, Q. Mou, M. Sun, et al., Cancer theranostic nanoparticles self-assembled from amphiphilic small molecules with equilibrium shift-induced renal clearance. Theranostics, 2016, 6(10): 1703-1716.

[33] 33. Y. Yan, Z. Xiao, Y. Song, et al., ${ }^{99} \mathrm{mTc}-\mathrm{HYNIC-MPG:}$ A novel SPECT probe for targeting mutated EGFR. Bioorganic \& Medicinal Chemistry Letters, 2015, 25(7): 1647-1652.

[34] Z. Xiao, Y. Song, W. Kai, et al., Evaluation of 99mTcHYNIC-MPG as a novel SPECT radiotracer to detect EGFR-activating mutations in NSCLC. Oncotarget, 2017, 8(25): 40732-40740.

[35] S. Wang, P. Huang, and X. Chen, Stimuli-responsive programmed specific targeting in nanomedicine. ACS Nano, 2016, 10(3): 2991-2994.

[36] M. Hou, P. Xue, Y.E. Gao, et al., Gemcitabinecamptothecin conjugates: a hybrid prodrug for controlled drug release and synergistic therapeutics. Biomaterials Science, 2017, 5(9): 1889-1897.

[37] D. Cui, L. Ma, X. Zhi, et al., Advances and prospects of nanotheranostic technology for gastric cancer. Nano Biomedicine \& Engineering, 2016, 8(4): 219-239.

Copyright $\subseteq$ Baozhong Shen. This is an open-access article distributed under the terms of the Creative Commons Attribution License, which permits unrestricted use, distribution, and reproduction in any medium, provided the original author and source are credited. 